\title{
Research on Applications of ARMA in Forecasting of
}

\section{Time Series}

Ruohong Li, School of Mathematics and Statistics, Beijing Institute of Technology, Beijing, 102488

\begin{abstract}
ARMA (Autoregressive moving averagemodel)is a linear prediction model using to predict the short-term value. This paper analyzes the concept and features of ARMA, and then uses it to predict the sea wave signals. From the experimental results, the prediction is quiet effective.
\end{abstract}

KEYWORDS:forecasting of time series; ARMA

\section{Introduction}

A lot of data in real life are appeared in the form of time series. The time series is an important kind of complex data objects, regardless of changes in a stock price of a commodity. The numbers of sunspots monthly sales or natural areas, precipitation and so on are shaped into a time sequence. In the social, economic, technological and other fields there exist a lot of time series data needs to be further analyzed and processed, people hope that through the analysis of these time series data found that the development and change law of a certain phenomenon from as much as possible, thus the extraction of accurate information they need, to predict the future use of these data, thus to better control the purpose of future events. Scientific prediction is that it has scientific basis, including the theory, data, calculation method, and other factors, depend on the knowledge of the objective law and master. The purpose of prediction is to provide objective basis for the formulation of the plan and decision of economy, many fields of engineering are inseparable from the forecast, predict daily weather, network traffic prediction, forecast the trend of stock are closely linked with our daily life. The current forecasting method is not mature enough. Some of the major natural disasters, such as earthquake, tsunami, have not formed an effective method of forecasting. To solve the problem of prediction is the key to the intrinsic law of development through the study of historical data make clear the sequence and dependence, thus by using time series to forecast the future of its change rule. And the reality of data structure changes, to explore the essential form of historical data, to create a truly predictable timing model is a great challenge. Autoregressive moving averagemodel (ARMA) supposes a set of time series of the future value of linear phase on its historical valuefor stationary sequences. The predicted wave signal is a basic issue in the prediction of ship motions.If the sea wave can be effectively predicted, short-time prediction of ship motion is very useful. Therefore, this paper uses ARMA to predict wave signal time series.

\section{Principles of ARMA Prediction}

The model of ARMA (auto regressive moving average model) is the method of parameter methods of high resolution spectrum analysis method. This method is a typical method of stationary random process rational spectrum, applicable to a large class of practical problems. It is better than the AR model method and MA model method, which has the spectrum, and the spectral resolution performance is 
excellent estimation more accurate. However, its parameters estimation more complicated.

Autoregressive moving average model $\operatorname{ARMA}(p, q)$ expression is:

$$
x_{t}-\phi_{1} x_{t-1}-\phi_{2} x_{t-2}-\ldots-\phi_{p} x_{t-p}=a_{t}-\theta_{1} a_{t-1}-\ldots-\theta_{q} a_{t-q}
$$

With the delay operator can be type called:

$$
\phi(B) x_{t}=\theta(B) a_{t}
$$

are Autoregressive and moving average coefficients.

In this, $\phi(B)=1-\phi_{1} B-\ldots-\phi_{p} B^{p}$ p order

autoregressive coefficient polynomial

$$
\theta(B)=1-\theta_{1} B-\ldots-\theta_{q} B^{q}
$$

is q order autoregressive coefficient polynomial.

In that, $\mathrm{p}, \mathrm{q}$ are the autoregressive part and the moving average part of the order.

$$
\begin{array}{r}
\phi_{i}(i=1,2, \ldots p), \theta_{j}(j=1,2, \ldots q) \\
R_{k}=\phi_{1} R_{k-1}+\ldots+\phi_{p} R_{k-p}+R_{x a}(k)-\theta_{1} R_{x a}(k-1)-\ldots-\theta_{q} R_{x a}(k-q) . \\
\text { self-correlation function is }
\end{array}
$$

In that

$$
\begin{aligned}
& R_{x a}(k)=0, k>0, \\
& R_{x a}(k) \neq 0, k \leq 0 .
\end{aligned}
$$

It can be judged according to the expressions of the autocorrelation function, $\operatorname{ARMA}(\mathrm{P}, \mathrm{q})$ unlimited trailing autocorrelation function model, and AR (P) model of the same, the exponential decay or oscillation. Through the calculation can also learned that ARMA (P, q) of MA property and partial correlation function of the model (q) is the same of the infinite tail.

$\operatorname{ARMA}(\mathrm{P}, \mathrm{q})$ order method and AR model $(\mathrm{P})$ to determine the order of the criteria as following description, ARMA (P, q) concrete form of model order determination criterion.

(1) AIC Criterion

We use ARMA (p, q) the time series $\left\{x_{t}, 1 \leq t \leq N\right\}$ to fit the model. The AIC function is defined as:

$$
\operatorname{AIC}(p, q)=N \lg \hat{\sigma}_{a}^{2}+2(p+q+2)
$$

(2) BIC Criterion

We use ARMA ( $p, q)$ the time series $\left\{x_{t}, 1 \leq t \leq N\right\}$ to fit the model. The BIC function is defined as:

$$
B I C(p, q)=N \lg \hat{\sigma}_{a}^{2}+(p+q) \lg N
$$

$\mathrm{BIC}$ is compatible, but it requires a larger sample size. Otherwise, the effect is not good.

The parameters moment estimation of the model of ARMA (p,q)is :

$$
\hat{\sigma}_{a}^{2}=\frac{1+\hat{\phi}_{1}^{2}+\ldots \hat{\phi}_{p}^{2}}{1+\hat{\theta}_{1}^{2}+\ldots+\hat{\theta}_{q}^{2}} \hat{\sigma}_{x}^{2}
$$

\section{Solutions to Problems of Accounting Measure based on Fair Value}

We use MATLAB toolbox in $\mathrm{m}=\operatorname{armax}(\mathrm{x}$, $[\mathrm{p}, \mathrm{q}]$ ) model parameter estimation function. $\mathrm{X}$ 
is used for observation sequence prediction, also need to use iddata function is transformed into the time series structure required; $\mathrm{P}, \mathrm{q}$ for the ARMA model of autoregressive order and the moving average order. The first 2944 data selected wave signals do the 20 step forecast, forecast of 2945th to 2964 data. ARMA prediction model: A (q) y (T) $=\mathrm{C}(\mathrm{q})$ e ( $\mathrm{t}$ ). ARMA this time predicted by the established model and the prediction results are as follows $(\mathrm{p}=8, \mathrm{q}=6)$ :

$$
\begin{gathered}
A_{(q)}=1-7.315 q+23.94 q^{2}-45.77 q^{3}+55.92 q^{4}-44.7 q^{5}+22.83 q^{6}-6.813 q^{7}+0.9093 q^{8} \\
C_{(q)}=1-0.717 q+1.873 q^{2}-0.4154 q^{3}+1.188 q^{4}-0.06823 q^{5}+0.4643 q^{6}
\end{gathered}
$$

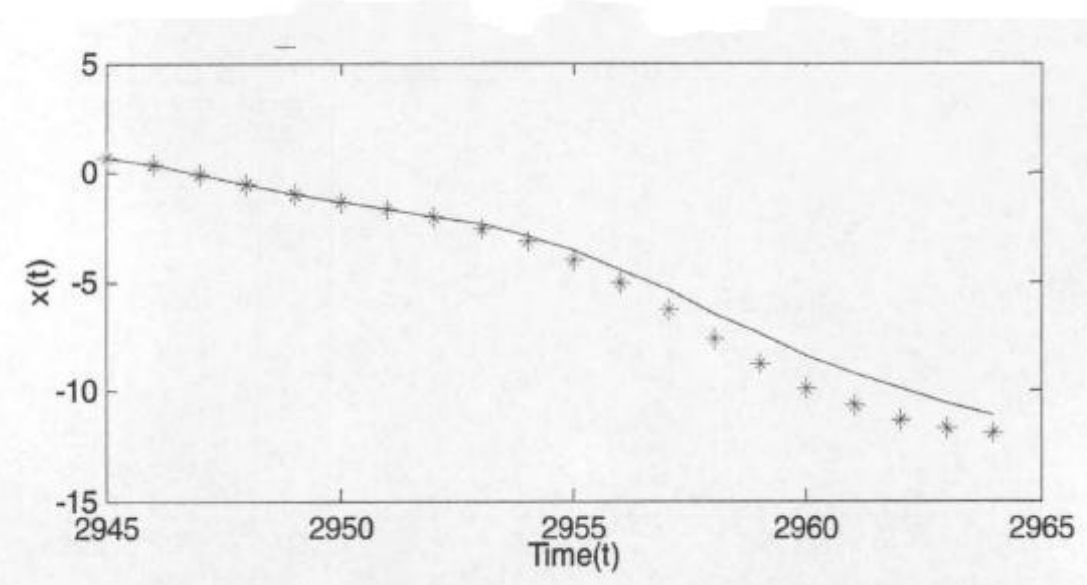

Figure 1. Comparison between the Observed Value and the Prediction Value based on AMRA (* represents the prediction value, the other represents the observed value)

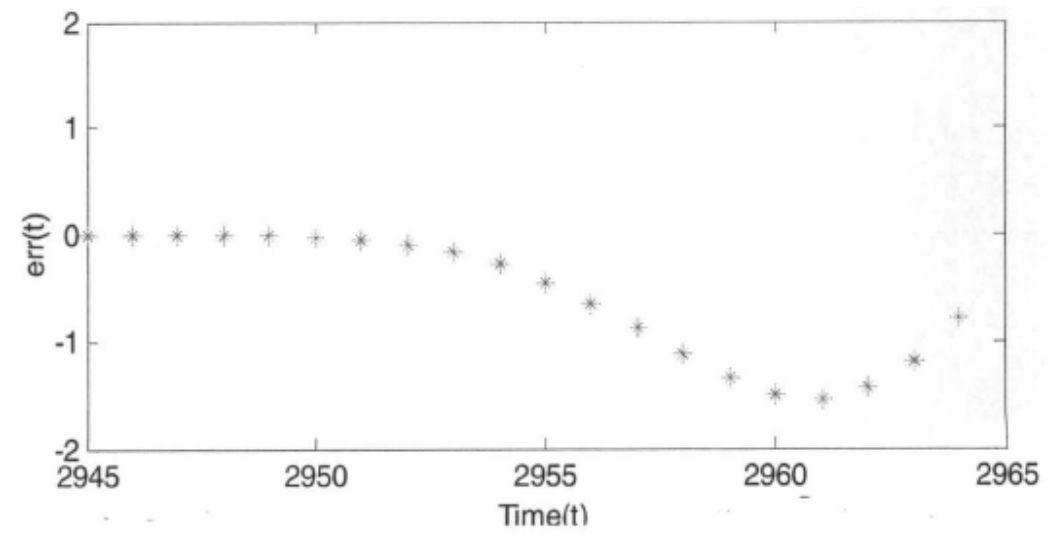

Figure 2. Error Value based on AMRA 


\section{CONCLUSIONS}

At present, the time series prediction method mainly includes to set up the construction of the nonlinear model and linear model. The linear model is adopted in autoregressive moving average (ARMA) model. The most popular is to establish the nonlinear model of neural network prediction, of whichapplications of BP neural network and RBF neural network is popular. Although the steps of the linear model for ARMA prediction cannot achieve very long, the short-term prediction of sea wave signal is very effective.

REFERENCE:

[1] Martin, R.J. A metric for ARMA processes [J]. Browse Journals \& Magazines, 2012, 27

[2] Swami, A. A unified approach to modeling multichannel ARMA processes[J]. Browse Conference Publications, 2011, 38

[3] Md.Kamrul. An Approach to ARMA Model Identification from Noise Corrupted Output Measurements[J]. Transactions on Fundamentals of Electronics, 2012, 18 